\title{
L'Impact de la Microfinance, où en sommes-nous? Une Revue des Méthodes utilisées dans les Évaluations Empiriques
}

\author{
Gnoudanfoly A. Soro \\ UFR des Sciences Économiques et de Gestion (UFR-SEG), \\ Enseignant-Chercheur \\ Université Félix Houphouët Boigny de Cocody-Abidjan-Côte d’Ivoire
}

\section{Résumé}

Quel est impact réel de la microfinance? Réduit-elle vraiment la pauvreté ? Deux principales interrogations récurrentes auxquelles les praticiens du microcrédit sont confrontés. Les derniers évènements sur la crise de la microfinance en Inde en 2011 ont renforcé le scepticisme des opposants sur les réelles capacités du microcrédit à venir à bout de la pauvreté dans le monde. De nombreuses initiatives ont été entreprises pour vérifier son impact à travers le monde. Les résultats de ces études restent mitigés et semblent remettre en doutes la rhétorique de de la microfinance comme un outil efficace de lutte contre la pauvreté. Le présent papier est une revue des travaux empiriques de l'impact de la microfinance présentée sous l'angle de l'évolution des méthodes d'évaluation. Tout en mettant relief les limites des méthodes d'évaluation, l'analyse met relief l'importance des évaluations en microfinance.

Mots clés: Microfinance, Evaluation d'Impact, Approche non-expérimentale, Évaluation aléatoire, Biais de sélection. 


\title{
The Impact of Microfinance, Where Do We Stand? A Review of Methods Used in Empirical Evaluations
}

\author{
Gnoudanfoly A. Soro \\ UFR des Sciences Économiques et de Gestion (UFR-SEG), \\ Enseignant-Chercheur \\ Université Félix Houphouët Boigny de Cocody-Abidjan-Côte d’Ivoire
}

\begin{abstract}
What is the real impact of microfinance? Does it really reduce poverty? These are two basic recurring questions faced by microcredit practitioners. The latest events on the microfinance crisis in India in 2011 have strengthened opponents' skepticism about the real capabilities of microcredit to overcome poverty in the world. Numerous studies have been conducted to verify its impact worldwide. The results of these studies remain mixed and seem to cast doubt on the rhetoric of microfinance as an effective instrument to fight against poverty. This paper is a review of empirical studies of the impact of microfinance presented in terms of evolution of evaluation methods. While highlighting the limitations of evaluation methods, the analysis highlights the importance of assessments on microfinance.
\end{abstract}

Keywords: Microfinance, Impact Evaluation, Non-Experimental Approach, Randomized Evaluation, Selection Bias

\section{Introduction}

L’idée que la microfinance est un instrument de lutte contre la pauvreté a été largement discutée dans la littérature. Après plus de trois décennies d'expérimentation, les résultats de l'impact de la microfinance ne semblent pas faire consensus entre praticiens, chercheurs et spécialistes de la microfinance. Cette situation soulève la problématique de l'évaluation de l'impact des programmes de développement, plus spécifiquement de la microfinance. Les approches et méthodes d'évaluation sont au centre des préoccupations, notamment en ce qui concerne leurs pertinences dans les processus d'évaluation. La question fondamentale à laquelle tente de répondre toute évaluation d'impact est de savoir comment les conditions de vie des bénéficiaires diffèrent de celles qui auraient prévalu si le programme n’avait pas été mis en œuvre. En d'autres termes, quelle serait la situation des bénéficiaires en l'absence du programme? Ou quelle aurait été la situation de 
ceux qui n’ont pas bénéficié du programme s’ils y avaient été exposés? La solution idéale consisterait à comparer le ou les outcomes d'un même individu selon qu'il bénéficie ou non du programme dont on mesure l'impact. Or cette situation ne peut s'observer en même temps sur un même individu car, soit il bénéficie du programme ou soit il n'en bénéficie pas. On pourrait aussi apprécier la situation de l'individu avant et après la mise en œuvre du programme. Mais, cette analyse est insuffisante ou du moins ne prouve pas grand-chose, car la situation de l'individu entre ces deux périodes pourrait être influencée par des facteurs autres que le programme. Il ne suffit pas non plus de comparer simplement les moyennes des variables de résultats à un moment donné ou dans le temps entre individus bénéficiaires et non bénéficiaires d'un programme et attribuer cette différence aux effets du programme. En effet, il existe des différences entre les individus, car ceux qui bénéficient du programme n'ont pas forcément les mêmes caractéristiques sociodémographiques que ceux qui n'en profitent pas. Une autre difficulté réside dans le choix des critères de sélection de la clientèle propres à chaque institution de microfinance(IMF). En effet, ces critères peuvent induire des différences de caractéristiques entre les personnes éligibles et non éligibles au microcrédit. Ces différences systématiques entre les individus créent un biais de sélection dans le processus d'évaluation. Les facteurs qui contribuent à la présence de biais sont multiples et constituent des limites à la pertinence des évaluations.

Une des solutions largement utilisée consiste à considérer un groupe d'individus exposé au programme et un autre groupe similaire d'un point de vue des caractéristiques observables et non exposé au programme. Le groupe exposé au programme est défini comme le groupe de traitement et le groupe non exposé, le « contrefactuel » ou le groupe de contrôle. La difficulté réside dans le contrôle à la fois de l'hétérogénéité sur les variables observables et sur les inobservables. Pour résoudre ces problèmes de biais de sélection et d'hétérogénéité, diverses techniques statistiques et économétriques sont mises en œuvre dans la démarche d'évaluation qui découlent de deux principales approches méthodologiques: l'approche non expérimentale et l'approche expérimentale ou randomisation. Mais quelle est la pertinence de ces approches dans l'analyse de l'impact de la microfinance ? Laquelle de ces deux approches semble être la plus pertinente dans l'évaluation des programmes et projets de microfinance ? Quel est l'impact ou quels sont les effets qui découlent de ces analyses de l'impact de la microfinance ? Cette étude tente de répondre à ces interrogations. Dans cette logique, l'objectif de cette analyse est double, elle vise d'une part, à conduire une réflexion critique sur les approches et méthodes d'évaluation, et d'autre part, à repérer l'impact et les effets réels de la microfinance. Pour ce faire, nous mobilisons des travaux empiriques pour analyser l'impact de la microfinance, à partir, d'une 
part, de l'approche non expérimentale et d'autre part, de l'approche expérimentale. Nous supposons que la pertinence de chaque approche est liée au contexte, aux types et aux méthodes de collecte des données et des méthodes mobilisés dans le processus d'évaluation. De plus, nous supposons que l'impact et les effets présupposés de la microfinance sur les pauvres restent mitigés et l'idée qu'elle réduit la pauvreté est difficilement prouvée empiriquement. Notre démarche méthodologique procède par une revue des méthodes d'évaluation à partir d'études empiriques ayant mobilisées chacune de ces approches.

Il faut noter que ce travail ne s'inscrit pas dans une analyse technique des outils et méthodes qui ont fait l'objet d'analyse dans divers travaux (Karlan, 2001; Kono and Takahashi, 2010; L'Horty and Petit, 2010; Soro, 2019). La suite de ce travail appréhende l'évolution de l'analyse de l'impact de la microfinance sous deux angles d'approches: expérimentale et non expérimentale. Les résultats des études empiriques sont exposés, tout en mettant relief les méthodes mobilisées et leurs limites dans les évaluations de la microfinance. Ainsi, nous exposons successivement une revue de travaux empiriques qui mobilisent l'approche non expérimentale (Section 2), ensuite, quelques travaux s'inscrivant dans l'approche expérimentale (Section 3), et nous concluons.

\section{Une revue de l'impact en microfinance par l'approche non expérimentale}

L’approche non expérimentale de l'évaluation regroupe des méthodes statistiques et économétriques qui utilisent des bases de données dont les enquêtes n'ont pas suivi un processus aléatoire de choix des enquêtés. En général, les méthodes d'évaluation utilisées sont des méthodes quantitatives ${ }^{4}$.

\section{Première étude scientifique rigoureuse}

Les premières études académiques qui ont tenté d'étayer ou de réfuter par des arguments scientifiques l'impact prétendument positif de la microfinance concernaient des évaluations non expérimentales. Parmi ces travaux académiques, l'étude de Pitt and Khandker (1998) réalisée au Bangladesh est l'une citées dans les travaux de la microfinance. Elle semble même constituer le point de départ de la justification ou de l'identification rigoureuse de l'impact en microfinance. Par la suite, ce travail a fait l'objet de critiques et a stimulé la publication de plusieurs travaux sur le secteur du microcrédit. Ces critiques sont diverses et portent tant sur les résultats, les hypothèses que sur les outils statistiques que les auteurs ont utilisés. Ces

\footnotetext{
${ }^{4}$ Les méthodes couramment utilisées sont les scores de propensions, la méthode des variables instrumentales, la méthode de régression discontinue et la méthode de la différence double, etc.
} 
auteurs ont collecté des données dans des villages ayant reçu des programmes de microfinance et dans d'autres villages où il n'y avait pas de programmes. Ils tiennent compte des effets supposés fixes entre villages de sorte à atténuer les biais de localisation du programme. Pour évaluer l’impact, le critère d’éligibilité utilisé est un facteur exogène à la participation au programme de microcrédit, à savoir la nécessité de détenir moins d'une acre de terre. Ce critère suppose qu'il n'existe pas de transactions foncières fréquentes ou régulières entre les ménages. L’outil statistique appliqué ${ }^{5}$ est une régression discontinue associée à une technique économétrique complexe avec un seuil critique, la possession par le ménage d'une acre de terre ${ }^{6}$. En termes de résultats d’impact sur les bénéficiaires, les auteurs trouvent que le microcrédit a un effet plus important sur le comportement des ménages lorsque ce sont les femmes qui participent au programme. Un autre résultat intéressant de cette étude est qu'un emprunt de $100 \mathrm{taka}^{7}$ contracté par une femme permet d'augmenter la consommation annuelle du ménage de 18 taka. En outre, ils constatent qu'un accroissement de 1 \% du crédit octroyé par la Grameen Bank à une femme augmente en moyenne le taux d'enrôlement scolaire des filles de $1,86 \%$, ce qui prouve l'effet significatif et positif du crédit sur la scolarisation des enfants et en particulier des filles. Ainsi, ils estiment que l'intervention des IMF permettrait chaque année à 5 \% des bénéficiaires de sortir de la pauvreté (Gubert et Roubaud, 2005).

\section{Critiques de l'étude de Pitt et Khandker (1998)}

Les critiques portées à l'encontre de cette étude ne se sont pas faites attendre. Les premières viennent de Morduch (1998) qui réfute les hypothèses énoncées par Pitt et Khandker (1998) et propose de reprendre autrement leurs estimations. À partir de la méthode de la double différence, il obtient des résultats distincts de ceux de Pitt et Khandker $(1998)^{8}$, et impute ces différences de résultats au fait que le marché foncier du Bangladesh est un marché très actif où les clients vendent et achètent des lopins de terre. En tenant compte de cette réalité, le critère d'éligibilité au microcrédit basé sur la possession de moins d'une acre de terre de Pitt et Khandker (1998) n'est pas approprié. De plus, il considère que ce critère d'éligibilité n’est pas conforme aux pratiques réelles de la Grameen Bank qui, en réalité, offre aussi des crédits à des personnes qui possèdent plus d'une acre de terre. Ce qui met en doute le

${ }^{5}$ Weighted Exogenous Sampling Maximum Likelihood-Limited Information Maximum Likelihood-Fixed Effects (WESLML-LIML-FE). Pour plus de détails voir Pitt et Khandker (1998).

${ }^{6}$ Une acre est la mesure d'une surface agraire. Elle varie selon les pays. En France, une acre correspond à 52 ares et en Angleterre à 40,47 ares.

${ }^{7}$ Un Taka $(B D T) \stackrel{ }{0} 0,0137$ Dollars USD contre 11 taka.

${ }^{8}$ Pitt et Khandker (1998) ont utilisé la méthode de régression discontinue. 
critère d’éligibilité et donc les résultats des analyses de Pitt et Khandker (1998). En termes de résultats d'impact, il conclut qu'il existe très peu d'effets positifs sur les bénéficiaires du programme. Toutefois, il constate également comme eux, un lissage de la consommation des bénéficiaires.

\section{Des critiques aux perspectives d'amélioration de l'analyse de l'impact}

D’autres auteurs après Morduch (1998), Mckernan (2002), Menon (2006), Roodman et Morduch (2009) ont formulé d'autres critiques à l'encontre de l'étude de Pitt et Khandker (1998). En se basant sur une méthodologie similaire à celle de ces auteurs, Mckernan (2002) examine la sensibilité des estimations liées au critère d'éligibilité. Il constate que les effets de la participation au programme sont peu sensibles au critère d'éligibilité (moins d'une acre de terre) pour les clients de la Grameen Bank et aucune influence pour les clients des autres $\mathrm{IMF}^{9}$. Les ménages à haut rendement sont les plus susceptibles de participer au programme de microcrédit de la Grameen Bank. De plus, il constate que la méthode utilisée ne permet pas de contrôler le biais de sélection car, il produit une surestimation des profits des entreprises de plus de $200 \%$.

Face aux diverses critiques portées à leur étude de 1998, Khandker (2005) va tenter d’apporter des réponses en utilisant des données de panel. Il associe à la base de données de 1991-1992 une seconde constituée entre 1998 et 1999. Ces deux bases de données lui permettent de répondre à certaines critiques de Morduch (1998). Il justifie son choix des données de panel en trois raisons essentielles. D’abord, les résultats obtenus à partir de données en coupe transversale ne sont pas robustes. Ainsi, la fiabilité et la robustesse des résultats de l'étude sont mises en doutes. En outre, certaines études en coupes transversales ont montré que la mesure de l'impact dépend fortement des méthodes utilisées pour traiter la question de l'endogénéité du programme (Lalonde, 1986) ${ }^{10}$. L'endogénéité peut être liée à divers facteurs, notamment l'emplacement et la participation au programme de microfinance. Ainsi, serait-il judicieux, selon lui, d'apprécier la robustesse des résultats avec une méthode autre que celle de Pitt et Khandker (1998). Ensuite, en utilisant les données de panel, les effets fixes entre ménages sont moins tributaires du critère d’éligibilité qui est lié au titre foncier des bénéficiaires du programme de microcrédit. Enfin, les données en coupe transversale ne fournissent que des effets de court terme du programme. Or, en réalité, un programme met du temps avant d'influencer certaines variables telles que les actifs, l'investissement dans le capital humain ou l'éducation des enfants. L'usage des données de panel permet de prendre en compte cette réalité et de mesurer

${ }^{9}$ Il s'agit notamment de la Bangladesh Rural Advancement Committee (BRAC) et de la Bangladesh Rural Development Board's (BRDB).

${ }^{10}$ Cité par Khandker (2005). 
les effets à long terme du programme. Globalement, les résultats de cette nouvelle évaluation donnent un impact positif sur les clients, mais sont sensiblement inférieurs à ceux de l'étude précédente. La baisse de l'impact sur les clients s'expliquerait, d'une part, par le fait que le modèle révisé réduit l'impact de la microfinance et, d'autre part, par le fait que les clients avaient des rendements marginaux des emprunts décroissants sur la période ${ }^{11}$ (Karlan et Goldberg, 2007). Ces rendements des emprunts des femmes sont passés à $10,5 \%$ avec l'estimation en données de panels contre $18 \%$ pour l'estimation en coupe transversale. La participation au programme de microfinance aurait fait chuter la pauvreté modérée de 8,5 \% et l'extrême pauvreté de 18,2 \% sur la même période (Khandker, 2005). De plus, la participation des femmes au programme de microfinance a eu un impact positif sur la consommation contrairement à celle des hommes pour lesquels l'auteur ne trouve aucune incidence. Un impact positif est également constaté sur la santé et l'éducation des enfants lorsque le prêt est contracté par une femme. Pour un emprunt de 100 taka par une femme, l'impact augmente de 20,5 taka contre 18 taka dans l'étude de Pitt et Khandker (1998). Ce résultat est obtenu par addition des effets des deux périodes correspondant à 4,3 taka pour les emprunts en cours et 16,2 taka pour les emprunts passés (Karlan et Goldberg, 2007).

Une des critiques qui remet en doute les résultats de cette nouvelle étude est le taux d'attrition non négligeable entre les deux périodes que Morduch (1998) avait déjà dénoncé. En effet, environ 30 \% des ménages de l'enquête de 1991-1992 n'étaient plus membres entre 1998 et 1999. Ce taux d'attrition pourrait s'expliquer par la faillite de ses membres qui pour diverses raisons n’ont pas su gérer leurs emprunts.

L'effet positif du lissage de la consommation des ménages constatés dans les études de Pitt et Khandker (1998) et Morduch (1998) a été vérifié par Menon (2006) avec les mêmes données d'enquête de 1991-1992. Elle construit un échantillon de 8 Grameen thanas pour analyser l'impact du lissage de la consommation. Elle conclut que même si le microcrédit aide à améliorer la capacité des clients à lisser leur consommation face aux chocs saisonniers, ses effets tendent à disparaître après quatre ans de participation au programme. Les effets du prêt atteignent un maximum après deux années d'adhésion puis déclinent par la suite.

Les mêmes données de Pitt et Khandker (1998) vont être utilisées par Chemin (2008) avec une méthode d'appariement par les scores de propension pour analyser l'impact du microcrédit autrement. Il détermine l'effet moyen du traitement sur les dépenses de consommation par habitant et sur d'autres indicateurs (taux de scolarisation des filles et des garçons, l'offre de main d'œuvre féminine et masculine, etc.). Il observe un impact positif de la

\footnotetext{
${ }^{11}$ Une période d'environ 7 ans.
} 
microfinance sur les dépenses des ménages bénéficiaires, sur l’offre de maind'œuvre et sur la scolarisation des filles et des garçons. De plus, les ménages bénéficiaires dépensaient en moyenne $3 \%$ de plus que les ménages non bénéficiaires. En outre, il constate que la microfinance a plutôt un effet de lissage du revenu qu'un lissage de la consommation comme suggéraient Morduch (1998) et Pitt et Khandker (1998).

Toujours à la recherche d'une meilleure compréhension de l'impact de la microfinance suite aux divergences constatées dans les études précédentes, Roodman et Morduch (2009) décident de revisiter les résultats de Pitt et Khandker (1998), Morduch (1998) et de Khandker (2005). Pour ce faire, ils utilisent la base de données originale des études précédentes pour reconstruire les données de leur étude. Les tests statistiques montrent bien que les données reconstruites correspondent bien à celles utilisées par ces études. Une fois les données reconstruites, ils utilisent les mêmes méthodologies d'estimation de sorte à répliquer les résultats de Pitt et Khandker (1998) et de Khandker (2005), puis ils procèdent à des tests spécifiques. Ils constatent avec surprise que les résultats de l'exercice de réplication et de tests spécifiques donnent des résultats différents de ceux des études précédentes. Ils trouvent que l'impact du microcrédit sur les bénéficiaires dans les trois études est très faible et parfois peu fiable. L'impact sur la consommation est négatif et significatif contrairement aux résultats de Pitt et Khandker (1998). En outre, ils constatent que la volatilité de la consommation est beaucoup plus faible en comparaison aux résultats de Morduch (1998). Ils émettent des réserves sur les résultats obtenus, notamment ceux attestant que le microcrédit a amélioré la situation des personnes extrêmement pauvres. Par ailleurs, ils notent que le passage aux données de panels proposé par Khandker (2005) n'apporte pas nécessairement une estimation crédible, car sa méthode ne parvient pas à compenser l'absence de variations exogènes dans l'utilisation du microcrédit. Ils ne concluent pas à un effet négatif ou nul du microcrédit sur les femmes et leurs ménages, mais ils insistent plutôt sur les difficultés qu'il y a à analyser la relation causale de l'octroi de microcrédit et l'amélioration du bien-être des ménages par l'utilisation des méthodes non expérimentales. Ils suggèrent que la piste des expérimentations aléatoires soit davantage explorée.

Le besoin de réalisation d'évaluations moins coûteuses en temps et en argent a suscité dans le secteur de la microfinance la mise en œuvre de diverses méthodes d'évaluation, au nombre desquelles la comparaison pipeline ${ }^{12}$. Diverses études d'évaluation se sont appropriées cette méthode. C'est le cas de l'étude de Coleman (1999) réalisée en Thaïlande du Nord pour évaluer

${ }^{12}$ Encore appelée pipeline matching, elle consiste à considérer, d'une part les clients et les non clients d'une microfinance pour faire des estimations dites «naïves » et d'autre part, les clients anciens et nouveaux, pour analyser les possibles effets d'un programme de microfinance. 
l'impact sur les bénéficiaires des programmes de microcrédit et sur l'ensemble de la collectivité. L'enquête concernait les membres et les non-membres des villages de traitement ou des villages ayant participé au programme de microfinance, mais également les membres potentiels et les non-membres des villages de contrôle. Ces villages devraient participer un an plus tard au programme. Pour contrôler l'auto-sélection dans le programme, l'auteur utilise les clients actuels et les futurs clients et pour limiter le biais de localisation du programme, il prend en compte des non emprunteurs dans chaque village. La configuration des données collectées lui permet de réaliser une analyse par la méthode des doubles différences. En termes de résultats, son étude ne donne aucune preuve d'effets positifs ou négatifs des crédits sur les ventes, les économies, les actifs ou les dépenses d'éducation des ménages. Il constate plutôt des effets négatifs sur les dépenses médicales et l'augmentation des emprunts auprès des prêteurs informels. Cette situation s'expliquerait, selon lui, par le fait que de nombreux clients ont rejoint le programme de microfinance pour des raisons sociales. Ils constatent que nombreuses sont les personnes qui pour éviter d'être en marge du mouvement global de la collectivité se sont inscrites dans des groupes de prêts. Ces personnes contractent les prêts sans avoir préalablement identifié un projet viable dans lequel investir. Par conséquent, elles ont tendance à utiliser les prêts à des fins de consommation plutôt qu'à des activités productives. Quand vient le moment de rembourser, elles empruntent auprès des prêteurs informels. La participation au programme de microfinance devient néfaste pour ces personnes qui sont prises dans un cercle vicieux d'endettement. Cette situation serait plus préoccupante si la zone d'étude n'était pas couverte par d'autres institutions de microcrédit. En effet, dans les villages enquêtés, $63 \%$ des ménages étaient membres de la Banque de l'agriculture et des coopératives agricoles $\left(\mathrm{BAAC}^{13}\right)$. Cette présence semble avoir réduit l'endettement excessif des populations auprès des prêteurs informels dont les taux d'intérêt sont très élevés. Un autre résultat de cette étude est que les ménages qui avaient une dotation initiale importante sont ceux qui avaient une forte probabilité de participer au programme de microfinance et ils en profitaient mieux que les autres.

La même méthode a été expérimentée par Hulme et Mosley (1996) pour analyser l'impact du microcrédit dans sept pays. Globalement, on retient des conclusions de ces études que le microcrédit a un impact relativement positif sur les revenus des emprunteurs pauvres. Le microcrédit aurait permis une augmentation moyenne des revenus par rapport au groupe de contrôle allant de 10 à $12 \%$ en Indonésie et d'environ $30 \%$ au Bangladesh et en Inde ${ }^{14}$.

\footnotetext{
${ }^{13}$ Bank for Agriculture and Agricultural Cooperatives.

${ }^{14}$ Cité par Gubert et Roubaud (2005).
} 


\section{La prise en compte des biais possibles}

On reproche aux travaux précédents leurs négligences des possibles biais de sélection liés à la localisation des programmes. En effet, les différences de caractéristiques entre villages peuvent influencer l'offre et la demande de crédit. En plus de ces critiques, Morduch (1998) émet d'autres réserves notamment sur la similarité du groupe de contrôle et du groupe traité. La situation des membres des groupes de contrôle dès les débuts des programmes était très différente de celle des groupes d'emprunteurs. C'est le cas par exemple de la $\mathrm{BRI}^{15}$ en Indonésie où le différentiel de revenu entre les deux groupes était de 40 \% en moyenne au profit des emprunteurs. L'impact mesuré reflète donc l'écart de revenu entre les deux groupes et non l'effet du crédit (Gubert and Roubaud, 2005).

À la suite des critiques de Morduch (1998) et de Pitt (1999) d'autres critiques ont été formulées à l'encontre de la méthode de comparaison pipeline. C'est le cas des études de Karlan et Goldberg (2007), Tedeschi (2008), Tedeschi et Karlan (2010) qui dénoncent l'existence de biais de sélection liés à l'attrition, au temps, à la dynamique managériale et organisationnelle des institutions. En effet, il est possible de constater des abandons de clients après leur entrée dans le programme de microfinance. Ce biais devient important lorsque les abandons deviennent nombreux et cela peut mettre en doute les résultats de l'évaluation. En effet, des clients peuvent décider de quitter un programme parce qu'ils ont échoué dans leurs projets d'investissement, d'autres par contre, ont pu réussir à améliorer leurs activités et peuvent se passer des services de l'IMF ou décider de s'orienter désormais vers les banques formelles. Ignorer ou négliger ces abandons peut conduire à une surestimation ou une sous-estimation de l'impact du programme.

Un autre critique de cette approche est la difficulté de distinction des anciens et des nouveaux clients. L'hypothèse d'une grande similarité entre ces deux sous-groupes de clients est problématique. La distinction entre anciens clients et nouveaux clients est liée au choix de l'individu et à la méthode de sélection de la clientèle mise en œuvre par le programme. Diverses raisons motivent le choix ou non de participer en tant que client dès la mise en place des services d'une IMF. Par exemple, la prise de risque diffère d'une personne à une autre. Ainsi, dès le lancement d'un programme de financement, des personnes peuvent décider d'y participer aussitôt, tandis que d'autres attendront plus de temps pour diverses raisons liées à leurs attitudes risquophobes. Ces derniers prendront le temps d'observer la situation des premiers clients avant de s'engager éventuellement dans le programme. Pour d'autres, l'absence d'une occasion d'affaires ou de projets, l'obtention d'un emploi, des problèmes familiaux parfois contraignants peuvent limiter

\footnotetext{
${ }^{15}$ Bank Rakyat Indonesia.
} 
l'adhésion au programme. Pour les femmes, par exemple, les contraintes familiales liées à l'éducation des enfants, aux tâches ménagères parfois excessives sont difficiles à concilier avec la conduite d'activités productives. Ce qui limite parfois leur adhésion au microcrédit.

Par ailleurs, la dynamique d'expansion, la recherche de viabilité et de pérennisation des programmes des IMF ne sont pas prises en compte par ces études. En effet, pour certaines raisons, une IMF peut décider de s'installer dans une région plutôt que dans une autre. La plupart du temps, elles s'installent d'abord dans un environnement prometteur où elles peuvent avoir des clients solvables, généralement situés au-dessus du seuil de pauvreté. Elles ne se tournent vers les pauvres et les très pauvres qu'après acquisition d'une assise financière solide et une parfaite connaissance des pratiques commerciales et culturelles locales. Il se crée de facto une différence systématique entre les nouveaux et les anciens clients de l'institution.

Dans tous les cas précédents, il y a auto-sélection des clients invalidant l'hypothèse de similarité des caractéristiques entre anciens et nouveaux clients. Dans ce cadre, les résultats de l'évaluation de l'impact ne peuvent donc être portés uniquement à l'actif du programme de microcrédit.

Des études ont été faites pour tenter de prendre en compte les limites reprochées aux précédents travaux. C'est l'exemple de l'étude de Tedeschi (2008) sur le Pérou dans laquelle elle utilise deux séries de données de panel collectées auprès des ménages clients et non clients de la Mibanco, une IMF installée à Lima. L'étude tente d'apprécier les biais potentiels causés par les abandons, la durée de la sélection et la localisation du programme. La nature des données lui permet de faire une analyse relativement similaire à celle de Coleman (1999) à la différence qu'elle utilise des données de panel. Ces résultats montrent que la sélection est un problème important dans l'évaluation des programmes, car elle crée des différences entre les clients. Les emprunteurs potentiels ont des revenus sensiblement plus élevés que ceux qui ne seront pas emprunteurs. Ces résultats l'amènent à conclure que l'approche de comparaison pipeline surestime l'impact du programme de microfinance. Cette surestimation dépend fortement des problèmes d'attrition et de durée de la sélection. Bien que l'impact de la microfinance sur les bénéfices des entreprises reste significatif et positif, elle note que l'évaluation de l'impact par cette approche doit être appréhendée avec beaucoup de prudence.

Tedeschi et Karlan (2010) ont également tenté d'analyser les problèmes liés aux biais des programmes. Ils utilisent les mêmes données collectées par Tedeschi (2008) pour analyser les effets des abandons et du temps sur l'impact des programmes. Ils mettent en œuvre deux méthodes d'analyse: une analyse comparative de comparaison pipeline et une analyse en 
coupe transversale incluant les abandons ${ }^{16}$. Cette étude comme les précédentes met en évidence les effets des biais précités. Les auteurs concluent que la négligence ou l'ignorance des abandons surestime l'impact. Ils constatent par exemple, que le profit annuel des entreprises lucratives des clients était positif et augmentait de 4083 nuevos soles ${ }^{17}$ par an avec la méthode de comparaison pipeline. En revanche, lorsqu'ils prennent en compte les abandons, la baisse du profit est importante, soit environ 588 nuevos soles par an pour les mêmes entreprises. Les deux méthodes utilisées donnent des résultats différents lorsque l'on introduit les abandons.

Le problème de biais d'abandon est aussi souligné par Montgomery (2005) qui note que l'approche de comparaison pipeline ne permet pas de contrôler parfaitement ce type de biais. L'importance des abandons a également été souligné par Armendariz de Aghion et Morduch (2005) qui après révision d'un bon nombre d'études d'impact constatent que les taux d'abandon étaient significatifs allant de 3,5\% à $60 \%$ par an dans différents programmes de microfinance à travers le monde. Il faut noter que la question des abandons n'est pas spécifique à la méthode de comparaison pipeline, elle est transversale à toutes les méthodes d'analyse de l'impact qu'elles soient expérimentales ou non.

Suite aux critiques portées à son étude de 1999 notamment sur les problèmes liés à l'auto-sélection des membres des programmes de microcrédit dans les villages, Coleman (2006) va procéder à une autre évaluation sur la même base de données que son étude de 1999. Pour résoudre ce problème, il considère le pouvoir de décision ou la position de chaque membre au sein des banques villageoises comme une variable permettant de distinguer les membres du comité des membres ordinaires des banques villageoises. Comparant ses nouveaux résultats à ceux de son étude de 1999, il constate que les effets du microcrédit sur les membres ordinaires ne sont pas significatifs. Par contre, les effets sur les membres du comité sont positifs et significatifs à différents niveaux : sur le revenu, sur l'épargne, sur le temps de travail et les dépenses. Les membres du comité sont les dirigeants de la banque villageoise constitués du président, du vice-président et du trésorier. Ils représentent en réalité le segment relativement mieux lotis dans la société. Ces résultats sont liés aux différences entre les catégories de membres et aux comportements des membres du comité. En effet, les membres du comité utilisaient, par exemple plusieurs noms pour emprunter au point que le montant total de leurs emprunts correspondait parfois au montant total des emprunts des membres ordinaires. Il conclut que l'impact moyen négligeable du microcrédit obtenu dans son

${ }^{16}$ Ils nomment cette méthodologie: recalculated cross-sectional approach.

171 Peru nuevos soles $\simeq 0,352175$ USD. 
étude précédente est en partie dû au fait que le microcrédit ne profite pas réellement aux pauvres. Ce résultat est conforme aux problèmes récurrents évoqués dans la gestion des groupes ou des associations dans lesquels les dirigeants s'accaparent le plus souvent les financements reçus pour leurs activités au détriment des membres ordinaires.

Dans les zones rurales aux Philippines, Kondo et al. (2008) empruntent approximativement la même démarche méthodologique de Coleman (2006) pour évaluer l'impact du microcrédit dans 116 villages membres de 28 IMF de Philippines. Ils constatent que l'impact des prêts sur le revenu par habitant est positif et légèrement significatif. Cela est également vrai pour les dépenses alimentaires et les dépenses totales par habitant. Cependant, ils constatent que cet impact est négatif pour les ménages plus pauvres, et positif et croissant pour les ménages riches. Dans l'étude de Coleman (2006) comme dans celleci, les bénéfices du microcrédit sont accaparés par les ménages aisés au détriment des ménages pauvres.

À la différence des études précédentes, Takahashi et al. (2010) ont combiné les méthodes de doubles différences et d'appariement par les scores de propension pour analyser l'impact du microcrédit sur le bien-être des pauvres en Indonésie. L'avantage de leur méthode selon eux réside dans sa capacité à contrôler les biais résultant des caractéristiques observables par les scores de propension et les caractéristiques inobservables par la méthode des doubles différences. Le contrôle de ces biais, toutes choses égales par ailleurs permet d'obtenir des résultats très proches des effets réels du programme mis en œuvre. Ils montrent que l'impact du microcrédit sur le revenu des ménages et les profits des entreprises individuelles n'est pas significatif, tandis que l'impact sur les ventes de ces mêmes entreprises est positif et significatif. Des divers résultats obtenus, ils concluent que le microcrédit contribue à agrandir la taille d'une entreprise, mais pas son profit. En outre, si l'impact sur les ventes des entreprises individuelles est positif, ses effets ne concernent que les non pauvres. Les pauvres ne bénéficient du microcrédit que par l'accroissement de l'investissement en éducation de leurs enfants. Ils considèrent que si le microcrédit peut contribuer à la réduction intergénérationnelle de la pauvreté à travers l'investissement en éducation, l'impact ne saurait à l'évidence être immédiat.

Que retenir de ces évaluations non expérimentales? Il semble difficile de déduire que la microfinance a un impact nécessairement positif sur les bénéficiaires de ses services. Les résultats des évaluations sont mitigés et aucune méthode ne semble faire consensus. Des résultats positifs de l'impact de la microfinance obtenus dans certains travaux sont contredits par d'autres analyses, ce qui remet en cause les postulats de base de l'impact de la microfinance. 
En somme, deux remarques ressortent de ces évaluations. Les résultats des évaluations sont tributaires des méthodes d'évaluation. Le choix de ces méthodes est fonction d'une part, des données dont dispose l'évaluateur pour son analyse et, d'autre part, du type d'impact, de politique ou de programme objet de l'analyse.

Les méthodes d'évaluation non expérimentale connaissent toutes des limites quant à la suppression ou la réduction des biais. Les problèmes liés au biais de sélection, d'abandon, de contamination, de randomisation, et aux bases de données, au groupe de contrôle, etc. sont les limites des méthodes de cette approche (cf. tableau 1). Ces dernières années l'approche expérimentale est de plus en plus sollicitée pour évaluer l'impact des programmes et pour lever les limites de l'approche non expérimentale. Les évaluations par cette approche peuvent servir de complément à une évaluation non expérimentale. Ainsi, servent-elles comme moyen de vérification de l'impact d'un programme par comparaison de résultats expérimentaux et non expérimentaux. Mais, qu'en est-il des évaluations par l'approche expérimentale en microfinance? La section suivante présente brièvement l'approche expérimentale notamment celles des évaluations aléatoires en vogue aujourd'hui et quelques études de cas portant sur la microfinance.

\section{Les évaluations aléatoires}

La méthode des évaluations par assignation aléatoire est particulièrement récente en microfinance. Ce n’est que récemment que cette méthode vulgarisée par le $J-P A L$ a été utilisée dans le secteur de la microfinance par ses chercheurs ${ }^{18}$. D'un point de vue historique, la méthode issue des sciences médicales a été introduite en agronomie puis en sciences sociales. Les expérimentations aléatoires ou randomisées ont connu un intérêt croissant en économie du développement ces dernières années avec des auteurs comme Banerjee et Duflo (2009); Giné et Karlan (2008); Karlan et Zinman (2009a), etc. Les travaux qui ont utilisé cette méthode dans le secteur de la microfinance sont récents et peu nombreux à notre connaissance. Ces travaux ont produit des résultats intéressants qui ont permis l'expansion de certains programmes ou dans d'autres cas de l'intégration de services complémentaires à leurs activités principales. D’autres études ont proposé des préconisations pour l'adaptation ou la modification de l'offre de services. Les enseignements de ces études sont divers suscitant ainsi une sollicitation

${ }^{18}$ J-PAL: Jameel Poverty Action Lab est un Laboratoire d'action contre la pauvreté créé en 2003 au département d'économie du MIT (Massachusetts Institute of Technology). Depuis, il s'est développé en réseau mondial de professeurs unis par leur utilisation de l'évaluation aléatoire comme moyen de répondre à des questions critiques pour la lutte contre la pauvreté. La mission de J-PAL est d'aider à la réduction de la pauvreté dans le monde en faisant en sorte que les politiques mises en œuvre pour y parvenir soient fondées sur des preuves scientifiques. 
croissante des institutions de microfinance de taille importante et des ONG pour justifier à travers des analyses rigoureuses l'impact de leurs interventions auprès des bailleurs de fonds.

L'étude de Banerjee et Duflo (2009) ${ }^{19}$ est l'une des premières à avoir initié l'expérimentation randomisée pour évaluer un programme de microfinance ${ }^{20}$. En Inde, les auteurs choisissent de manière aléatoire la moitié des 104 bidonvilles de Hyderabad pour l'ouverture d'un programme de microcrédit. L’autre moitié ne bénéficiant pas du programme est prise comme groupe de contrôle. L'évaluation du programme aboutit à plusieurs résultats importants. Les auteurs montrent, entre autres résultats que les effets du microcrédit sont hétérogènes. En effet, ils constatent des modifications de comportement dans les dépenses de consommation des ménages. Ces dépenses pour les biens durables ont augmenté chez les ménages qui avaient une activité avant l'installation du programme de microfinance, mais celles de biens non durables n’ont pas connu de changements notables. Les ménages qui avaient une forte probabilité d'être propriétaires d'entreprises ont augmenté leurs dépenses de consommation de biens durables et ont réduit leur consommation de biens non durables. À l'inverse, les ménages qui avaient une très faible probabilité d'être propriétaire ont augmenté leur consommation de biens non durables. Les ménages se mettaient à réduire les dépenses dites « inutiles » comme l'achat de thé ou de friandises. Le prêt contracté devant être remboursé, toutes les restrictions sont mises en œuvre pour réussir. Ils n'observent aucune transformation en termes d'acquisition de pouvoir ou de gestion de l'argent du ménage. Ils constatent également que les impacts moyens sur les dépenses par habitant, les dépenses d'éducation et de santé ne sont pas statistiquement significatifs. Sur une période de 15 mois, les familles qui avaient fondé une entreprise étaient passées de $5 \%$ à $7 \%$, ce qui n’était pas négligeable (Banerjee et al., 2011). Malgré ces résultats peu reluisants, les auteurs conclurent que la microfinance pouvait être reconnue comme un des instruments essentiels à la lutte contre la pauvreté. Toutefois, ils suggèrent d'autres évaluations afin d'observer les effets du programme sur le long terme.

Une autre étude similaire à la précédente a été réalisée par Crépon et al. (2008) pour analyser la réaction des populations des zones rurales du Maroc à l'introduction d'un nouveau programme de microcrédit de Al Amana, la principale IMF du pays. Ces auteurs réalisent une enquête sur un échantillon de 1550 ménages tirés au hasard dans 16 villages. Le groupe traité est tiré dans 8 villages et le groupe de contrôle est tiré dans les 8 autres villages. Une analyse des résultats de la participation au programme révèle que l'acceptation du programme est relativement faible. Plusieurs facteurs observables ont

\footnotetext{
${ }^{19}$ Banerjee et Duflo (2009) sont les initiateurs et vaillants défenseurs de la randomisation en microéconomie du développement.

${ }^{20} \mathrm{Il}$ s'agit du programme de la microfinance Spandana.
} 
influencé la participation au programme, notamment, le temps, la structure des prêts, les types d’activités et l’expérience des ménages dans leurs activités et dans l'utilisation du crédit. La structure des prêts semble mieux s’adapter aux activités non agricoles. Les ménages qui possèdent une expérience de crédit et une capacité d'endettement ont une probabilité forte de participer au programme de microcrédit.

L’expérimentation aléatoire a également été mise en œuvre par Karlan et Zinman (2009a) pour mesurer les effets de l'expansion du microcrédit aux micro-entrepreneurs de Manille. Ils montrent qu'il n’existe pas de différences statistiques entre le revenu du ménage, la probabilité d'être sous le seuil de pauvreté, la qualité de la nourriture et la probabilité de consulter un médecin en raison de contraintes financières entre les groupes traités et de contrôle. Toutefois, ils trouvent un effet du programme sur le genre contraire aux idées reçues. En effet, ils constatent que l'accès au crédit augmente les profits des micro-entrepreneurs hommes et non ceux des femmes. De plus, les hommes semblent plus disposés à utiliser leurs profits pour la scolarisation de leurs enfants que les femmes ${ }^{21}$. Toutefois, ils ne perçoivent pas de preuves d'accroissement du bien-être des ménages liées à l'accès au microcrédit, mais plutôt une diminution du bien-être subjectif des ménages membres. Globalement, les auteurs concluent que le fonctionnement du microcrédit se traduit par une amélioration de la gestion des risques et des investissements au niveau du ménage et non sur l'activité pour laquelle le crédit est demandé.

L'expérimentation aléatoire est également un moyen de tester des hypothèses, de validation ou de réfutation de théories dans différents contextes. Tel est le cas de l’étude réalisée par Giné et Karlan (2008) aux Philippines. L’expérimentation a porté sur le programme BULAK de la Green Bank of Caraga, une banque opérant dans le secteur rural. Ces auteurs ont testé l'hypothèse selon laquelle les prêts de groupe sont un moyen efficace de réduction de l'aléa moral tel que le prétend la théorie économique en microfinance. Ils choisissent de manière aléatoire la moitié des prêts de groupe de l'institution et les convertissent en prêts individuels. Après trois ans d'expérimentation, ils montrent que les prêts de groupe ne sont pas si importants dans la réduction de l’aléa moral. Toutefois, ils conviennent que leurs résultats doivent être appréhendés avec réserves, car les prêts convertis étaient initialement des prêts de groupe et cela a peut-être influencé leurs résultats. Ils n'arrivent donc pas à conclure avec certitude sur l’importance ou non du groupe dans l'élimination des mauvais risques.

${ }^{21}$ Il faut tout de même signaler que les hommes représentaient seulement environ 15 \% de l'échantillon de l'étude. 
De même, Karlan and Zinman (2009b) ont tenté d'analyser l'incompatibilité des taux d'intérêt élevés avec des crédits à la consommation octroyés aux pauvres dans une IMF en Afrique du Sud. L'expérimentation a consisté à mettre en œuvre une stratégie de relâchement de la contrainte de crédit qui pèse sur les emprunteurs marginalisés. Les emprunteurs marginalisés sont des personnes dont les demandes de crédit à la consommation ont été rejetées par l'IMF. Parmi ces personnes, ils en repêchent de manière aléatoire puis, leurs octroient des prêts d'une durée de 4 mois avec des taux d'intérêt de 11,75 \% équivalents à un taux annuel de 200 \%. Malgré ces taux d'intérêt élevés, les auteurs constatent, 6 à 12 mois après, que les bénéficiaires des prêts marginaux ont été les plus susceptibles de garder leurs emplois. Ils détenaient les revenus les plus élevés et étaient moins susceptibles de souffrir de la faim. Néanmoins, ce résultat ne leur permettait pas d'affirmer que les clients se sentiraient beaucoup mieux si les taux d'intérêts étaient plus bas. Ils constatent que ces taux en vigueur semblent bénéficier aux clients et les prêts accordés aux personnes marginalisées sont également rentables pour l'IMF.

Cette étude comme les précédentes illustre bien l'intérêt qu'il y a à construire des politiques sur la base des évaluations aléatoires. L'impact analysé est crédible, précis et est facilement compréhensible pour les décideurs politiques. Les résultats obtenus de ces évaluations remettent parfois en cause les postulats de bases de la microfinance et certaines théories économiques. Les caractéristiques des prêts, le type de programme évalué et le contexte environnemental et socioculturel peuvent conduire parfois à des résultats contraires aux objectifs ou à tout le moins inattendus des programmes. Toutefois, cette approche comme la précédente présente des limites. Les problèmes liés à l'éthique, aux problèmes de généralisation des résultats, à la contamination de l'échantillon, aux biais de substitution et de croisement, etc. constituent les limites des méthodes de cette approche ( $c f$. tableau 1). Par ailleurs, le coût de mise en œuvre de ces évaluations mobilise des ressources importantes comparées à l'approche non expérimentale.

Au terme de cette analyse de l'évaluation des programmes de microfinance, il apparaît que l'évaluation est fortement dépendante d'une part, de l'approche mise en œuvre et d'autre part, des outils méthodologiques utilisés par l'évaluateur. L'exigence de rigueur scientifique de ces approches nécessite des bases de données importantes dont la collecte demande du temps et des moyens financiers importants que la plupart des IMF ne peuvent pas financer. Que retenir comme approche pertinente ? L'analyse réalisée ne conduit pas à une réponse tranchée sur une méthode meilleure qu'une autre. Chaque approche et les méthodes qui en découlent ont aussi bien des points positifs que des limites non négligeables ( $c f$. tableau 1 ). Ce tableau révèle aussi que chacune des méthodes peut être adoptée selon les moyens financiers, le 
contexte et les objectifs de l'analyse de l’évaluateur. En outre, on s'aperçoit que dans un souci d'analyses scientifiques rigoureuses et pertinentes, la complémentarité des deux approches est nécessaire. En effet, certaines limites et lacunes de l'une sont comblées ou prises en compte par l'autre et vis-versa.

Qu'en est-il de l'impact et des effets de la microfinance ? Il ressort de cette revue empirique que l'impact prétendu de réduction de la pauvreté de la microfinance n’est pas prouvé dans les travaux sur l'impact. Certains effets positifs sur la consommation, l’éducation des enfants, la santé des ménages et l'empowerment sont parfois obtenus dans certaines études, tandis que d'autres perçoivent peu ou pas du tout d'effets directs et parfois même indirects sur les bénéficiaires. Le tableau 2 en annexe, fait un résumé des principaux travaux utilisés dans ce travail. Comme on peut le constater dans la dernière colonne de ce tableau, il est difficile de conclure à un impact et à des effets positifs de la microfinance sur les bénéficiaires. Les effets semblent varier selon le contexte, la région ou le pays, le type de programme et de services.

\section{Conclusion}

Comme toute politique de développement l’impact de la microfinance peut concerner plusieurs dimensions, notamment les effets du programme sur les bénéficiaires. Une connaissance de ces effets est indispensable pour une meilleure orientation du programme afin d'atteindre l'amélioration du bienêtre des bénéficiaires. On observe également que les résultats sont sensibles aux méthodes d’évaluation. Après plus de trois décennies, il ne semble pas se dégager un consensus sur l'impact de la microfinance tels qu'énoncés par postulats de base, lutte contre la pauvreté et amélioration du bien-être des populations bénéficiaires. Des études qui ont apporté un certain crédit empirique à ces postulats ont été au cours de l'évolution des outils d'évaluation remis en doute par de nouvelles études. Faut-il remettre en cause les méthodes d'analyse ou faut-il admettre que les effets de la microfinance restent mitigés sur les bénéficiaires? Pour que l'impact et les effets de la microfinance soient reconnus de tous, au moins de manière globale, il est nécessaire qu'il y ait un consensus sur les méthodes d'évaluation qui peuvent générer les résultats d'impact pertinents et fiables. À travers ce survol, on comprend mieux que les success stories des clients, les best practices d'IMF et les mérites de la microfinance présentés çà et là dans les forums et sommets internationaux par les praticiens, le plus souvent soutenus les gouvernants des pays en développement et par les partenaires au développement sont à prendre avec beaucoup de prudence. Par ailleurs, il convient de noter qu'à l'instar des autres secteurs de l'économie, l'évaluation constitue l'un défi majeur des programmes de développement. Ces évaluations sont indispensables d'une part, pour en tirer les leçons des bonnes pratiques et des échecs antérieurs, afin d'améliorer les programmes en cours et futurs, et d'autre part d’apprécier 
l'impact des programmes sur les bénéficiaires. Ce travail ne prétend pas à l'exhaustivité, l'analyse de l'impact des programmes connaît un foisonnement d'outils et de méthodes qui, nous semble-t-il, ne sauraient être répertoriés dans une seule analyse. Toutefois, nous avons mis un accent particulier sur les travaux académiques récents qui ont fait l'objet de reconnaissances scientifiques au vu de l'ampleur de leur citation dans les articles scientifiques dans le secteur de la microfinance.

\section{References:}

1. Armendariz de Aghion, B. and Morduch, J. (2005), The Economics of Microfinance, The MIT Press, Cambridge, Massachusetts London.

2. Banerjee, A. V and Duflo, E. (2009), “L’approche expérimentale en économie du développement”, Revue d'économie Politique, Vol. 119 No. 5, pp. 691-726.

3. Banerjee, A. V and Duflo, E. (2012), Repenser La Pauvreté, Édition: Seuil, Paris.

4. Charpail, C., Klein, T. and Serge, Z. (2005), "Evaluation des politiques d'emploi: la deuxième génération des Panels des bénéficières”, DARES, Ministère des affaires sociales, du travail et de la solidarité,France Document de Travail.

5. Chemin, M. (2008), "The Benefits and Costs of Microfinance: Evidence from Bangladesh”, Journal of Development Studies, Vol. 44 No. 4, pp. 463-484.

6. Coleman, B.E. (1999), "The impact of group lending in Northeast Thailand”, Journal of Development Economics, Vol. 60, pp. 105141.

7. Coleman, B.E. (2006), "Microfinance in Northeast Thailand: Who Benefits and How Much ?", World Development, Vol. 34 No. 9, pp. 1612-1638.

8. Crépon, B., Devoto, F., Duflo, E. and Parienté, W. (2008), "Pauvreté, accès au crédit et déterminants de la participation à un nouveau programme de micro crédit dans les zones rurales du Maroc", Document de Travail.

9. Fay, R.G. (1996), "Enhancing the Effectiveness of Active Labour Market Policies”, OECDE Labour Market and Social Policy Occasional Papers $N^{\circ} 18$.

10. Giné, X. and Karlan, D.S. (2008), "Peer Monitoring and Enforcement: Long Term Evidence from Microcredit Lending Groups with and without Group Liability”, World Bank, Mimeo.

11. Gubert, F. and Roubaud, F. (2005), "Analyser l'impact d'un projet de microfinance: L'exemple de l'Adéfi à Madagascar”, Agence Française de Développement, Document de Travail. 
12. Hulme, D. and Mosley, P. (1996), Finance Against Poverty, Volume 2., Routledge, London and New York.

13. Karlan, D.S. (2001), "Microfinance Impact Assessments: The Perils of Using New Members as a Control Group", Journal of Microfinance / ESR Review, Journal of Microfinance, Vol. 3 No. 2, pp. 75-85.

14. Karlan, D.S. and Goldberg, N. (2007), "Impact Evaluation for Microfinance: Review of Methodological Issues”, The World Bank, Doing Impact Evaluation Series No. 7.

15. Karlan, D.S. and Zinman, J. (2009a), “Observing unobservables: Identifying Information Asymetries with a Comsumer credit Field Experiment”, Econometrica, Vol. 77 No. 6, pp. 1993-2008.

16. Karlan, D.S. and Zinman, J. (2009b), "Expanding Microenterprise Credit Access: Using Randomized Supply Decisions to Estimate the Impacts in Manila”, Discussion Paper, Economic Growth Center, Yale University, Vol. 976, pp. 1-35.

17. Khandker, S.R. (2005), "Microfinance and Poverty: Evidence Using Panel Data from Bangladesh", The World Bank, Economic Review, Vol. 19 No. 2, pp. 263-286.

18. Kondo, T., Orbeta, A., Dingcong, C. and Infantado, C. (2008), "Impact of Microfinance on Rural Households in the Philippines", Nonie Working Paper 4.

19. Kono, H. and Takahashi, K. (2010), "Microfinance revolution: its effects, innovations, and challenges", The Developing Economies, Vol. 48 No. 1, pp. 15-73.

20. L’Horty, Y.L. and Petit, P. (2010), “Évaluation aléatoire et expérimentations sociales", Centre d'Études de l'Emploi, Document de Travail, Vol. 135 No. 12, p. 29.

21. Mckernan, S.-M. (2002), "The Impact of Microcredit Programs on Self-Employment Profits: Do Noncredit Program Aspects Matter?", The Review of Economics and Statistics, Vol. 84 No. 1, pp. 93-115.

22. Menon, N. (2006), "Non-Linearities in Returns to Participation in Grameen Bank Programs”, Journal of Development Studies, Vol. 42 No. 8, pp. 1379-1400.

23. Montgomery, H. (2005), "Meeting the Double Bottom Line: The Impact of Khushali Bank's Microfinance Program in Pakistan", Asian Development Bank Policy Papers No. 8.

24. Pitt, M.M. and Khandker, S.R. (1998), "The Impact of Group-Based Credit Programs on Poor Households in Bangladesh: Does the Gender of Participants Matter?", Journal of Political Economy, Vol. 106 No. 5, pp. 958-996.

25. Roodman, D. and Morduch, J. (2009), “The Impact of Microcredit on 
the Poor in Bangladesh : Revisiting the Evidence", Center for Global Development, Working Paper Series $N^{\circ} 174$.

26. Soro, G.A. (2019), "L'évaluation de l'impact en microfinance: Une revue des approches et méthodes d'évaluation”, À paraître in European Scientific Journal.

27. Tedeschi, G.A. (2008), "Overcoming Selection Bias in Microcredit Impact Assessments: A Case Study in Peru”, Journal of Development Studies, Vol. 44 No. 4, pp. 504-518.

28. Tedeschi, G.A. and Karlan, D.S. (2010), "Cross Sectional Impact Analysis: Bias from Dropouts”, Perspectives on Global Development and Technology. 
Tableau 1: Résumé des avantages et limites des approches

\begin{tabular}{|c|}
\hline Approche non expérimentale \\
\hline Points positifs \\
\hline $\begin{array}{l}\text { Résultats: Elle permet de déterminer les } \\
\text { différences moyennes des résultats entre } \\
\text { bénéficiaires et non bénéficiaires, mais aussi } \\
\text { l'effet marginal sur les bénéficiaires. } \\
\text { Connaissance : Elle permet de renforcer et } \\
\text { d'améliorer les institutions évaluées. } \\
\text { Coût : Coût relativement inférieur aux } \\
\text { évaluations aléatoires. }\end{array}$ \\
\hline $\begin{array}{c}\text { Points faibles ou limites } \\
\end{array}$ \\
\hline $\begin{array}{l}\text { 1. Estimations diverses : Les méthodes } \\
\text { d'estimation sont diverses et difficiles à } \\
\text { comprendre pour le décideur. De plus, les } \\
\text { résultats peuvent varier en fonction de la } \\
\text { spécification du modèle surtout lorsque la } \\
\text { théorie propose plusieurs alternatives. } \\
\text { 2.Complexité : L'usage de techniques } \\
\text { économétriques variées requiert des } \\
\text { hypothèses fortes sur la distribution du terme } \\
\text { d'erreur. } \\
\text { 3. Effets d'entrée du programme: Cela se } \\
\text { produit lorsque la population inscrite dans le } \\
\text { programme et le groupe de contrôle ne sont } \\
\text { pas représentatifs de la population qui serait } \\
\text { touchée si celui-ci était un programme national } \\
\text { en cours. }\end{array}$ \\
\hline
\end{tabular}

4. Le problème du groupe de contrôle : Les estimations sont sensibles à la sélection du groupe de contrôle est sélectionné. Il est souvent difficile d'obtenir un bon groupe de comparaison dans les enquêtes générales, notamment lorsque le programme porte sur un petit groupe dont la représentativité est très faible dans l'échantillon.

5. Biais de contamination: II est fonction des données du groupe de comparaison. Les individus peuvent avoir reçu un traitement similaire au groupe de contrôle qui n'est pas pris en compte par la base de donnée utilisée par l'évaluateur. Par exemple, les individus du groupe de contrôle peuvent avoir reçu des prêts autres que ceux du programme sur la même période. Si cette distinction n'est pas prise en compte on a un biais de contamination.

6. Base de données: En général, les bases de données ne sont pas construites dans l'optique de conduire une évaluation d'impact. Ainsi, l'évaluateur ne fait qu'à adapter les données à la situation par l'usage de variables proxy. Toutefois, les caractéristiques individuelles Approche expérimentale ou randomisation Points positifs

Résultats : Précision et pertinences des résultats de l'évaluation et facilité d'interprétation et de compréhension. Élimination du biais de sélection ; Connaissance du terrain d'étude; Production des données par l'évaluateur.

Connaissances : elle permet d'améliorer la qualité des services et d'adapter les services aux besoins des populations.

Points faibles ou limites

1. Éthique en question : La limitation de l'accès à un service utile au bien-être des populations érigées en groupes de contrôle en raison de l'évaluation

2.Problèmes de mise en œuvre : La méthode est difficile à mettre en œuvre dans une institution où les individus sont réfractaires aux changements d'habitude et plus facile dans le cadre de nouveaux programmes.

3. Incertitude de la période d'évaluation: II est difficile de savoir combien de temps peut prendre un programme pour modifier les comportements et donc de déterminer le temps nécessaire pour conduire une évaluation.

4.Contamination du traitement: L'évaluateur peut être tenté d'inscrire autant de personnes que possible dans l'expérience. Ce qui peut rendre le groupe différent de celui qui aurait été choisi en l'absence de traitement.

5. Expériences multiples: Plusieurs évaluations sont nécessaires pour comprendre et mieux adapter les service d'un programme. Par exemple, une première évalue l'impact et une seconde analyse les services à mettre en place.

6. Auto-sélection: Les problèmes d'autosélection se produisent lorsque le choix d'être traité ou non ou d’être bénéficiaire ou non n'est pas aléatoire et dépend du choix des personnes.

7. Équilibre partiel et généralisation L'impact obtenu est partiel et non global. La généralisation des résultats de micro-dispositifs est difficile dans la mesure où le système des marchés et des prix diffère d'une zone à une autre.

8. Contamination de l'échantillon: Elle se produit lorsque les personnes inscrites dans le traitement sont différentes de ce qu'est le groupe en l'absence de traitement.

9. Coût : Elles sont coûteuses en temps, en argent et en ressources humaines.

10. Les résultats: L’effet mesuré est la différence moyenne entre les bénéficiaires et les non bénéficiaires et non la distribution de l’impact. 
sont le plus souvent détaillées et permettre une évaluation.

7. Biais de sélection : Le biais de sélection est aléatoire et il existe une incertitude sur sa taille. Il est donc difficile de savoir quel est son niveau d'influence sur le résultat.

8. Des ajustements non expérimentaux: On a parfois recours à des ajustements non expérimentaux pour corriger certains problèmes. C'est le cas des problèmes d'attrition qui peuvent rendre l'échantillon moins représentatif de la population initiale à l'origine de l'expérimentation.

9. Biais de randomisation : II englobe un certain nombre de biais (sélection de site, les abandons ou les décrocheurs, et l'effet Hawthorne).
11. Biais de croisement: II survient lorsque le groupe de contrôle se confond avec le groupe de traitement ou se retrouve à un moment donné dans la zone du traitement. Cela peut entraîner des risques de contamination.

12.Effet d'entrée du programme: II se produit lorsque la population inscrite dans le programme et le groupe de contrôle n'est pas assez représentatif si on l'on veut étendre le programme à l'échelle nationale.

13.Biais de substitution: Il se produit lorsque le groupe de contrôle participe à un programme similaire comparable à celui reçu par le groupe de traitement.

Source: : Auteur adapté, à partir de Charpail et al. (2005) et Fay (1996)

Tableau 2: Résumé de quelques études

\begin{tabular}{|c|c|c|c|c|}
\hline Études & Régions & Objectifs & $\begin{array}{c}\text { Approche \& } \\
\text { Méthodes }\end{array}$ & $\begin{array}{l}\text { Impact sur les } \\
\text { bénéficiaires }\end{array}$ \\
\hline $\begin{array}{l}\text { Pitt et Khandker } \\
\text { (1998) }\end{array}$ & $\begin{array}{l}\text { Bangladesh/ } \\
\text { Asie du Sud }\end{array}$ & $\begin{array}{l}\text { Évaluer } \\
\text { l’impact du } \\
\text { microcrédit }\end{array}$ & $\begin{array}{l}\text { - A.N.E } \\
\text { - Régression } \\
\text { discontinue } \\
\end{array}$ & $(+)$ \\
\hline Morduch (1998) & $\begin{array}{l}\text { Bangladesh/ } \\
\text { Asie du Sud }\end{array}$ & $\begin{array}{l}\text { Évaluer } \\
\text { l’impact du } \\
\text { microcrédit }\end{array}$ & $\begin{array}{l}\text { - A.N.E } \\
\text { - Double } \\
\text { différence }\end{array}$ & $\begin{array}{r}\quad(+/-) \\
\text { - Effets mitigés } \\
\text { - - Lissage de la } \\
\text { consommation } \\
\end{array}$ \\
\hline Khandker (2005) & $\begin{array}{l}\text { Bangladesh/ } \\
\text { Asie du Sud }\end{array}$ & $\begin{array}{l}\text { Évaluer } \\
\text { l’impact du } \\
\text { microcrédit }\end{array}$ & $\begin{array}{l}\text { - A.N.E } \\
\text { - Données de } \\
\text { panels } \\
\end{array}$ & $\begin{array}{l}\text { Effets positifs, mais } \\
\text { faible, }\end{array}$ \\
\hline $\begin{array}{c}\text { Roodman et } \\
\text { Khandker(2009) }^{\circ}\end{array}$ & $\begin{array}{l}\text { Bangladesh/ } \\
\text { Asie du Sud }\end{array}$ & $\begin{array}{l}\text { Évaluer } \\
\text { l’impact du } \\
\text { microcrédit }\end{array}$ & \begin{tabular}{|l} 
- A.N.E \\
- Méthodes \\
identiques à \\
Pitt et \\
Khandker \\
(1998) et \\
Khandker \\
(2005), plus \\
application de \\
test spécifiques \\
\end{tabular} & \begin{tabular}{l}
\multicolumn{1}{c}{$(+/-)$} \\
- Effets très faibles et \\
peu fiables. \\
- Effet négatif sur la \\
consommation
\end{tabular} \\
\hline Coleman (1999) & $\begin{array}{l}\text { Thaïlande du } \\
\text { Nord/ Asie du } \\
\text { Sud }\end{array}$ & $\begin{array}{l}\text { Évaluer } \\
\text { l’impact du } \\
\text { microcrédit }\end{array}$ & $\begin{array}{l}\text { - A.N.E } \\
\text { - Pipeline } \\
\text { comparison } \\
\text { - Double } \\
\text { différence }\end{array}$ & $\begin{array}{l}\text { (-) } \\
\text { - Effets négatifs sur } \\
\text { dépenses de santé, } \\
\text { - Hausse de l'emprunt } \\
\text { informel }\end{array}$ \\
\hline Coleman (2006) & Idem & Idem & $\begin{array}{l}\text { - Idem, mais, ici } \\
\text { Prise en } \\
\text { compte de } \\
\text { l’auto-sélection }\end{array}$ & \begin{tabular}{l}
\multicolumn{1}{c}{$(+/-)$} \\
- Effet positif sur \\
d'autres clients et non \\
significatifs sur les \\
clients ordinaires \\
\end{tabular} \\
\hline Chemin (2008) & Idem & Idem & $\begin{array}{l}\text { - Pipeline } \\
\text { comparison }\end{array}$ & \begin{tabular}{l}
\multicolumn{1}{c}{$(+)$} \\
- Effet positif sur la \\
consommation,
\end{tabular} \\
\hline
\end{tabular}




\begin{tabular}{|c|c|c|c|c|}
\hline & & & $\begin{array}{l}\text { - Méthode } \\
\text { d’appariement- } \\
\text { scores } \\
\text { d’appariement }\end{array}$ & $\begin{array}{l}\text { - Effet positif sur la } \\
\text { scolarisation des filles } \\
\text { et des garçons, } \\
\text { - Lissage du revenu et } \\
\text { non de la } \\
\text { consommation. }\end{array}$ \\
\hline Tedeschi (2008) & $\begin{array}{c}\text { Pérou/Amérique } \\
\text { du sud }\end{array}$ & $\begin{array}{l}\text { Analyse de } \\
\text { biais } \\
\text { potentiels } \\
\text { (abandon, } \\
\text { durée, etc.) }\end{array}$ & $\begin{array}{l}\text { - A.N.E } \\
\text { - Données de } \\
\text { panels } \\
\text { - Double } \\
\text { différence }\end{array}$ & \begin{tabular}{l}
\multicolumn{1}{c}{$(+)$} \\
- Effet significatif \\
positif, mais doit être \\
pris avec réserves \\
compte tenu des biais.
\end{tabular} \\
\hline $\begin{array}{l}\text { Kondo et } \\
\text { al(2008) }\end{array}$ & $\begin{array}{c}\text { Philippines/Asie } \\
\text { du Sud Est }\end{array}$ & $\begin{array}{l}\text { Évaluer } \\
\text { l’impact du } \\
\text { microcrédit }\end{array}$ & $\begin{array}{l}\text { - A.N.E } \\
\text { - Double } \\
\text { différence }\end{array}$ & \begin{tabular}{l}
\multicolumn{1}{c}{$(+/-)$} \\
- Effet positif, mais peu \\
significatif; \\
- Effets négatif pour les \\
plus pauvres et positif \\
et croissant pour les \\
riches.
\end{tabular} \\
\hline $\begin{array}{c}\text { Banerjee et } \\
\text { Duflo (2009) }\end{array}$ & $\begin{array}{l}\text { Inde/Asie du } \\
\text { Sud }\end{array}$ & $\begin{array}{l}\text { Évaluer } \\
\text { l’impact d'un } \\
\text { programme de } \\
\text { microfinance }\end{array}$ & $\begin{array}{l}\text { - A.E. } \\
\text { - Randomisation } \\
\text { - Combinée aux } \\
\text { méthodes } \\
\text { économétriques }\end{array}$ & \begin{tabular}{l}
\multicolumn{1}{c}{$(+)$} \\
- Effets hétérogènes, \\
- Effet sur la \\
consommation, \\
- Effets faibles, mais \\
considère le \\
microcrédit comme \\
un instrument qui \\
peut contribuer à \\
réduire la pauvreté
\end{tabular} \\
\hline $\begin{array}{l}\text { Crépon et } \\
\text { al(2008) }\end{array}$ & $\begin{array}{c}\text { Maroc/Afrique } \\
\text { du Nord }\end{array}$ & $\begin{array}{c}\text { Analyser } \\
\text { l'impact de } \\
\text { l'introduction } \\
\text { d'un nouveau } \\
\text { programme de } \\
\text { microcrédit } \\
\end{array}$ & $\begin{array}{l}\text { - A.E } \\
\text { - Randomisation } \\
\text { et méthodes } \\
\text { économétriques }\end{array}$ & $\begin{array}{l}\text { (+) } \\
\text { - Participation au } \\
\text { programme faible ; } \\
\text { - Impact donc faible. }\end{array}$ \\
\hline $\begin{array}{c}\text { Karlan et } \\
\text { Zinman (2009a) }\end{array}$ & $\begin{array}{c}\text { Manille- } \\
\text { Philippines/Asie } \\
\text { du Sud }\end{array}$ & $\begin{array}{l}\text { Mesurer les } \\
\text { effets de } \\
\text { l’expansion } \\
\text { du microcrédit } \\
\text { aux } \\
\text { entrepreneurs }\end{array}$ & $\begin{array}{l}\text { - A.E. } \\
\text { - Randomisation } \\
\text { combinée à une } \\
\text { analyse } \\
\text { économétrique }\end{array}$ & $\begin{array}{l}\text { (-) } \\
\text { - Baisse du bien-être } \\
\text { subjectif des ménages, } \\
\text { - Gestion des risques et } \\
\text { des investissements } \\
\text { améliorés au niveau } \\
\text { des ménages et non } \\
\text { pour l'activité financé } \\
\text { par le prêt. }\end{array}$ \\
\hline $\begin{array}{c}\text { Karlan et } \\
\text { Zinman (2009b) }\end{array}$ & $\begin{array}{l}\text { Afrique du } \\
\text { Sud/Afrique }\end{array}$ & $\begin{array}{l}\text { Analyse des } \\
\text { taux d'intérêt } \\
\text { sur la } \\
\text { demande de } \\
\text { prêts à la } \\
\text { consommation } \\
\text { des pauvres } \\
\end{array}$ & $\begin{array}{l}\text { - A.E. } \\
\text { - Randomisation } \\
\text { combinée à une } \\
\text { analyse } \\
\text { économétrique }\end{array}$ & $\begin{array}{l}\quad(+) \\
\text { - Effets positifs sur les } \\
\text { clients marginalisés, } \\
\text { - Analyse insuffisante } \\
\text { pour juger l'effet du } \\
\text { taux d’intérêt. }\end{array}$ \\
\hline
\end{tabular}

Source : Auteur.

Note : Les effets énumérés pour chaque étude ne sont pas forcément exhaustif. A.N.E : Approche Non Expérimentale, A.E : Approche Expérimentale. 